\title{
MULTI-CRITERIA DECISION MAKING IN DESIGN OF PRINTED WIRE BOARDS
}

by

Y. Tokat $^{1}$, O. Palusinski ${ }^{2}$, and F. Szidarovszky ${ }^{1}$

\begin{abstract}
This paper presents an application of multi-criteria decision making in system level design of printed wire boards. The main decision variable is the number of signal layers, and the criteria being considered are the cost, electrical performance, and reliability. The weighting method is applied to find the most satisfactory solution. In the numerical example a complete description of the solution is given in terms of the weights or importance factors. The numerical results also show the stability of the solution.
\end{abstract}

1 Department of Systems and Industrial Engineering, University of Arizona, Tucson, Arizona 85721, USA.

2 Department of Electrical and Computer Engineering, University of Arizona, Tucson, Arizona 85721, USA 


\section{Introduction}

Packaging considerations in system design come into play when logical design is accomplished and there is a need to: (1) partition the system into chips, (2) decide about chip packaging, (3) decide about technology for second and /or higher level packaging. Several factors such as cost of packaging, electrical performance, reliability, mechanical performance etc. have to be taken into account in the decision process. Very often, these factors are in conflict with each other. For example improving the electrical performance as expressed by the speed of the system operation is achieved by miniaturization of packaging which yields shorter signal transmission paths and consequently shorter delays. However, this improvement is accompanied by the increase of dissipated power density and more advanced manufacturing technologies required to produce miniaturized packaging. The increased power density will cause the system components to either operate at higher temperature which will reduce their reliability or it will require better cooling which in turn will increase the system cost. The demand for advanced manufacturing technology will increase the cost and may reduce the system reliability. In general a package performs the following four functions: (a) mechanical support and protection from the environment, (b) power supply, (c) cooling (removal of heat generated by active circuits), (d) input/output connections and signal distribution. Metrics indicating how well these functions are performed by the package are often in conflict with each other as indicated by the above example. In addition improvements in performance indicators are typically associated with the cost increases.

A methodology for solving the conflicts in package performance indicators which appear in the design will be presented in this paper and will be illustrated by an example of system level considerations in selection of printed wire board (PWB) technology to house a specified number of modules (chip carriers). The major simplifying assumption is that the board is to be populated by a fixed number of identical modules. Extension to more general cases involving different modules with different numbers of input-output connections is being developed and will be presented in the future.

The most popular and most frequently used methods in multi-criteria decision making are based on the introduction of a single utility function which summarizes the different criteria into a single objective function. One important method of this kind is the weighting method. If $\mathrm{n}$ denotes the number of criteria and $Y_{i}(i=1,2, \ldots, \mathrm{n})$ denote the criteria, then the utility is defined as $\alpha_{1} Y_{1}+\alpha_{2} Y_{2}+\ldots+\alpha_{n} Y_{n}$, where the $\alpha_{i}$ weights are the importance factors of the criteria. It is usually assumed that these weights are all nonnegative and their sum equals one. A usual motivation for this method is explained in the following. We ask the decision maker to divide the $100 \%$ overall importance in between the different criteria, and the given portions are selected as the weights. If the criteria are given in different units, then it is hard to interpret the meaning of their weighted sum. In such cases, the criteria are first normalized into the unit interval by the linear transformation $\left(Y_{i}-\min \left\{Y_{i}\right\}\right) /\left(\max \left\{Y_{i}\right\}-\min \left\{Y_{i}\right\}\right)$ and the normalized criteria are multiplied by the weights and added up. Another type of utility based methods uses distances of vectors, and therefore these are called distance-based methods. In the applications of such methods the decision maker has to supply first an ideal point, 
coordinates of which are subjectively the "best" values of the different criteria. The feasible solution with the minimal distance from the ideal point is then accepted as the solution of the multi-criteria decision making problem. The solution depends on the distance function being selected. For a detailed description of utility-based methods and alternative techniques see for example Szidarovszky et al. (1986).

In this paper the weighting method is selected because of its simplicity. In the applications the most difficult issue is the selection of the weights. The decision makers usually do not have a firm quantifiable opinion about the relative importance of the different criteria, and therefore a sensitivity analysis must be performed to see how the solution depends on the particular weight selections. In our case study, we will give a complete description of the solution as the function of the weight selection to show which decision alternatives might become the solution and with what particular weight values. In addition, the stability of the solution is also quantified by the numerical results.

This paper is organized as follows. In Section 2, the problem formulation is given, Section 3 contains the details of the methodology being applied. The numerical results are presented in Section 4, and Section 5 includes the conclusions.

\section{Problem Formulation- Illustrative Example}

A high-speed, high-performance digital system composed of $N_{c}=100$ identical modules each requiring $N_{t}=200$ signal connections is to be packaged on a PWB. The module/PWB connections are to be made using surface mount technology. Given cost of a square inch of PWB we will determine the type of PWB as characterized by the wiring factor or the number of signal layers in the board structure in the case of given channel wiring density as determined by the via pitch and the number of conducting tracks in the channel. Considering the case of fixed channel wiring density, we relate the size of PWB to the number of signal layers using elementary wirability theory presented in [Johnson 1989, Palusinski 1997, Tummala and Rymaszewski 1989]. We will consider the cost of PWB, system electrical performance and reliability of the system in the board selection process.

\subsection{Cost Estimation}

The manufacturing cost can be estimated as proportional to the board area, thus it is given as

$$
Q=A q
$$

where $A$ is the board area [inch ${ }^{2}$ ], and $q$ is the unit cost of PWB [cost/ inch $\left.{ }^{2}\right]$. It is assumed for simplicity that

$$
A=P^{2} N_{c}
$$


where $P$ is the module pitch, and $N_{c}$ is the number of modules. The unit cost $q$ is estimated from the manufacturer data, and it depends on the number, $s$, of signal layers. The function

$$
q=0.06875 s^{2}-0.475 s+0.975 \quad\left[\$ / \text { inch }^{2}\right]
$$

is used in our study, as it approximates reasonably the low density curve corresponding to PWB's with low density of wiring shown in Figure 1. The cost data used in this analysis are higher than current market values as recently checked in a few points [Smith 1997]. However, the nature of dependence between the unit cost of PWB and the number of signal layers, which is important in the optimization methodology presented here remains the same. Because the current market data are not readily available to construct the appropriate relations, we decided to use the data given by Figure 1 for illustrative purposes in this study. In equation (2), $N_{c}=100$ is selected, and the module pitch, $P$, is determined from the wirability theory [Palusinski 1997, Tummala and Rymaszewski 1989]:

$$
\mathrm{P}=\frac{1}{s} \frac{\vartheta \mathrm{v}}{\mathrm{\kappa} n} N_{t}\left(1+0.1 \log N_{c}\right) N_{c}^{\gamma-0.5} \frac{p_{N}-1}{p_{N}}
$$

where $\vartheta=0.85$ is the empirical constant for average wire length formula, $\kappa=0.5$ is the wiring efficiency, $v=1.25$ [inch] is the via pitch, $n=3$ is the number of conducting tracks in a channel, $N_{t}=200$ is the number of signal terminals in the modules, $p_{N}=2$ is the average number of pins in the interconnection net. Furthermore $\gamma=0.55$ is the Rent's exponent.

Using equations (1)-(4) for all values of $s=2,3, \ldots, 16$, the second column of Table 1 representing the cost of appropriate boards is obtained.

\subsection{Estimating Electrical Performance}

The electrical performance $F$ is assumed to be the reciprocal of the clock cycle, $T_{c}$ :

$$
F=\frac{1}{T_{c}},
$$

where

$$
T_{c}=T_{p D}+T_{\text {skew }}+T_{\log i c}
$$

in the optimal case. The first term $T_{p D}$ is the delay due to packaging, $T_{\text {skew }}$ is the clock skew, and $T_{\log i c}$ is the delay of internal logic. In our analysis $T_{\text {skew }}$ is assumed to be equal 
to $T_{p D}$. The formula for packaging delay is derived under the assumption that the signal must travel across the board diagonal $(\sqrt{2} d)$ with $d=\sqrt{N_{c}} P$ being the board size dimension, the driver is matched to the line, the load of each receiver is represented by the discrete capacitor $c_{L}=2[p F]$.

Using the specified assumptions and delay definition, the package delay is determined as follows

$$
T_{p D}=2 \sqrt{2} d \tau+\eta \frac{Z_{0} c_{L}}{2} .
$$

The first term in the above formula is the line delay, where $\tau=33.3 \sqrt{4.6}[\mathrm{ps} / \mathrm{cm}]$ is the unit time delay of epoxy glass board lines, the second term reflects the distributed loads on the lines [Tummala and Rymaszewski 1989], $Z_{0}=50[\Omega]$ is the characteristic impedance, $c_{L}=2[p F]$ is the load capacitance, and $\eta$ is the number of discrete capacitors representing the receiver loads (number of taps). We select $\eta=\sqrt{2 N_{c}} / 3$, which corresponds to $1 / 3$ of modules along the board diagonal. The value of $T_{\log i c}$ is normally about $20 \%$ of clock cycle time. Therefore we represent it here with the average value of $T_{p D}$ for all considered values of $s$.

\subsection{Estimating Reliability}

The reliability of the system depends on the operating temperature. In order to depict the dependence between the reliability and the design parameter $(s)$, we built a relation between the dissipated power density, temperature and reliability. It is assumed that the parts involved are after the burn-in, the failure rate is constant, and thus the reliability can be easily calculated. The hazard rate depends exponentially on the temperature according to the Arrhenius law, and the temperature can be estimated on the basis of dissipated power density and average heat transfer coefficient. For this reason, we first estimate the power, $P_{w r}$, dissipated in the board using the following relation:

$$
P_{w r}=N_{c} \frac{\mathrm{E}}{T_{c}}=N_{c} \mathrm{E} F
$$

where, $\mathrm{E}$ is the energy dissipated per module during one clock cycle. An average value $\mathrm{E}=6 \cdot 10^{-8}[\mathrm{~J}]$ is selected in our analysis. This value is estimated for CMOS chips operating with $V_{L S}=5[\mathrm{~V}]$ voltage swing. As toward this end, we assume that there are $10^{15}$ gates of internal logic/ chip. A gate is loaded by a capacitor $C_{g}=10-100$ [fF]. The

energy need to switch a gate is estimated to be $\frac{1}{2} C_{g} V_{L S}{ }^{2}$ [Mead and Conway 1980]. 
Therefore, taking $C_{g}=100[\mathrm{fF}]$, we obtain $W_{g}=12.510^{-13}[\mathrm{~J}]$. Assuming that half of the gates switch per cycle we obtain the above given average energy dissipated per cycle.

The dissipated power density is computed as

$$
q_{s}^{\prime \prime}=\frac{P_{w r}}{A}
$$

and the temperature of chip active surface, which is approximately the same as junction temperature is given as

$$
T_{J}=T_{A}+\frac{q_{s}^{\prime \prime}}{h_{t}} .
$$

Here $T_{A}=300\left[{ }^{0} \mathrm{~K}\right]$ is the ambient temperature and $h_{t}$ is the average heat transfer coefficient which is assumed to be constant $\left(h_{t}=0.1\left[\frac{\mathrm{Watt}}{{ }^{\mathrm{o}} \mathrm{Kcm}^{2}}\right]\right)$. The hazard rate depends exponentially on the junction temperature according to the Arrhenius law:

$$
h\left(T_{j}\right)=h \cdot e^{\frac{E}{k}\left(\frac{1}{T_{1}}-\frac{1}{T_{J}}\right)},
$$

where $h=300 \cdot 10^{-9}\left[\frac{\text { failures }}{\text { hour }}\right]$ is the typical failure rate, at the junction temperature of $T_{1}=350\left[{ }^{\circ} \mathrm{K}\right]$ established under conservative operating conditions determined by low power density. The parameter, $E$, is the activation energy assumed as $E=1[\mathrm{eV}]$, and $k=8.6 \cdot 10^{-5}\left[\mathrm{eV} /{ }^{\mathrm{o}} \mathrm{K}\right]$ is the Boltzman constant.

Because of the assumption that the parts involved in the design are after burn-in (the failure rate is constant) the reliability can be calculated using the formula

$$
R=e^{-h\left(T_{J}\right) \cdot t}
$$

which relates the reliability to the selected here design variable.

For the purpose of this discussion, we consider a 1 year period: $t=24 \cdot 365[$ hours $]$.

\section{Methodology}

There are many approaches to solve multi-criteria decision problems. Because of its simplicity the weighting method is used in this study. In this method weights are assigned to each criteria and all of the criteria are merged into a single objective, which is 
then optimized.

\section{Example 1:}

In the first example, only two objectives are considered: cost and electrical performance. The weights are $\alpha$ and $1-\alpha$, respectively $(0 \leq \alpha \leq 1)$. The value $\alpha=0$ corresponds to the case, when only the second criterion is considered. If $\alpha=1$, then only the first criterion is considered. Equal importance of the criteria is modeled by the selection of $\alpha=1 / 2$. It is convenient to maximize both criteria, however in our case we want to minimize cost. Therefore, the cost data are multiplied by (-1) and the result is maximized. In order to avoid problems associated with addition of quantities of different units, we normalize both criteria into the unit interval $[0,1]$.The weighting method is then conveniently used with such normalized criteria.

There is a finite number of alternatives for the number of layers, $s=2,3, \ldots 16$. The normalized cost and electrical performance of each alternative have been calculated using the relations derived in the previous section. For each alternative, let $x_{i}$ be the normalized cost, and $y_{i}$ the corresponding normalized electrical performance. An alternative $\mathrm{r}$ is optimal among the $n$ discrete alternatives for a specific value of $\alpha$ if and only if

$$
\alpha x_{r}+(1-\alpha) y_{r} \geq \alpha x_{l}+(1-\alpha) y_{l}
$$

for all $l \neq r, l \in\{1, \ldots, n\}$. This relation can be rewritten as

$$
\alpha\left(x_{r}-y_{r}-x_{l}+y_{l}\right) \geq y_{l}-y_{r} \quad(\forall l \neq r)
$$

As we wish to give a complete description of the optimal solutions for all possible values of the weight $\alpha$, we proceed with the following algorithm:

Case 1: If $x_{r}-y_{r}-x_{l}+y_{l}>0$ then we consider two cases:

a)If $y_{l}-y_{r}>0$ then relation (14) holds if and only if

$$
\alpha \geq \frac{y_{l}-y_{r}}{x_{r}-y_{r}-x_{l}+y_{l}}
$$

b)If $y_{l}-y_{r} \leq 0$ then inequality (14) is always satisfied.

Case 2: If $x_{r}-y_{r}-x_{l}+y_{l}<0$ then we have the following two cases:

a)If $y_{l}-y_{r}>0$ then inequality (14) cannot be satisfied. That is, alternative $\mathrm{r}$ cannot be optimal for any value of $\alpha(0 \leq \alpha \leq 1)$.

b)If $y_{l}-y_{r} \leq 0$ then relation (14) holds if and only if 


$$
\alpha \leq \frac{y_{l}-y_{r}}{x_{r}-y_{r}-x_{l}+y_{l}} .
$$

Case 3: If $x_{r}-y_{r}-x_{l}+y_{l}=0$ then two sub-cases are examined:

a)If $y_{l}-y_{r}>0$ then relation (14) does not hold for any value of $\alpha(0 \leq \alpha \leq 1)$.

b)If $y_{l}-y_{r} \leq 0$ then inequality (14) holds for any $\alpha$ value $(0 \leq \alpha \leq 1)$.

After we compare alternative $r$ with all other alternatives, 1 , we can decide if alternative $r$ can be optimal and determine the range where it occurs. Since $\alpha \in[0,1]$ and inequalities (15) and (16) give upper and lower bounds for $\alpha$, for any given alternative $r$ we can easily decide if it is never optimal or obtain a closed interval for $\alpha$ when this alternative is optimal. These intervals will be called optimality intervals of the different alternatives. These intervals characterized by their end points and their lengths are of practical interest as explained below. Larger length shows that alternative $r$ becomes optimal more frequently with randomly selected weights(with uniform distributions). Larger length also shows that the solution is more stable in this region. When such comparisons are performed for all alternatives $r$, a complete characterization of the optimal solution is obtained.

Example 2:

In our second example we consider all three criteria. Similarly to the previous case, let $\alpha, \beta$, and $1-\alpha-\beta$ denote the weights, and $x_{i}, y_{i}$, and $z_{i}$ are the normalized criteria, where $x_{i}, y_{i}$ represent cost and electrical performance respectively, as previously defined, and $z_{i}$ denotes the reliability for alternative $i$. An alternative $r$ is optimal if and only if

$$
\alpha x_{r}+\beta y_{r}+(1-\alpha-\beta) z_{r} \geq \alpha x_{l}+\beta y_{l}+(1-\alpha-\beta) z_{l}
$$

for all $1 \neq \mathrm{r}$. This inequality can be rewritten as

$$
\alpha\left(x_{r}-z_{r}-x_{l}+z_{l}\right)+\beta\left(y_{r}-z_{r}-y_{l}+z_{l}\right) \geq z_{l}-z_{r} .
$$

Therefore, alternative $\mathrm{r}$ is optimal if and only if inequality (18) holds for all $1 \neq \mathrm{r}$, and

$$
\alpha \geq 0, \beta \geq 0, \alpha+\beta \leq 1
$$

Inequalities (18) and (19) are either contradictory, when alternative $r$ cannot be optimal for any $\alpha$ and $\beta$, or these linear inequalities determine a simplex in the twodimensional space, which will be called the optimality region for the different alternatives. Each such simplex can be characterized by its vertices, and by its area. Larger area shows that alternative $r$ becomes optimal more frequently with randomly selected weights (with uniform distributions), and it also shows that the solution is more stable in this region. 


\section{Numerical Results}

We have only one decision variable, which is the number of signal layers, $s$. The feasible alternatives are $s=2,3, \ldots, 16$. For each alternative we applied the method described in Section 2 to calculate the objective values.

\section{Example 1:}

The data set generated using previously described relations is given in Table 1 and illustrated in Figure 2. The first column lists the alternatives, the second, third and forth columns give the cost, electrical performance and reliability data, respectively. In the first example only cost and electrical performance are considered Thus in this example the last column is not considered. Before calculations, we first multiplied the cost data by (-1) in order to transform the problem to maximization. In addition, both the cost and electrical performance criteria were normalized as described in the introduction.

The first algorithm of the previous section was applied to these normalized data values. The results are given in Table 2, and illustrated in Figure 3. The first column shows that only alternatives $s=4, s=5$, and $s=16$ can be optimal. That is, $s=2, s=3$, and $s=6,7, \ldots, 15$ can never be optimal. The fact that alternatives $s=2$ and $s=3$ cannot be optimal is clear from Table 1, since for $s=4$ both objective function values are better. The criteria values for the 15 considered alternatives are illustrated in Figure 4. Each point corresponds to an alternative, the horizontal axis represents the normalized cost value and the vertical axis shows the normalized electrical performance. Each alternative $s=4,5, \ldots, 16$ can be considered as the best one in the sense that neither one of them can be improved in both criteria (in the multi-objective programming literature they are called the non-dominated solutions). In our method, a linear utility function is used, which can be represented as a straight line for each particular weight selection. Three such lines are shown in Figure 4, and the corresponding arrows indicate the directions in which these lines should be moved in order to improve the utility function values and to reach optimal solutions. The simple graphical representation shows that only alternatives $s=4,5$, and 16 can be optimal since the points corresponding to the alternatives $s=6,7, \ldots 15$ are below the line connecting the points representing alternatives $s=5$ and $s=16$. We observe here a similar phenomenon that occurs in non-convex continuous problems [Szidarovszky et al 1986].

\section{Example 2:}

In this case, all three criteria are included in the analysis. After multiplying the cost data by $(-1)$ and normalizing the criteria, the second method of the previous section was applied. The results are presented in Table 3 . The first column shows that only alternatives $r \geq 3$ can be optimal, $r=3$ has the largest and $r=6$ and $r=7$ have the smallest optimality areas. The optimality regions are illustrated in Figure 5. It is worth noting that, the optimality regions of alternatives 5-9 are very small and are located just above the 
optimality region of alternative 4 .

Notice that small length of optimality interval or small optimality area also show that in this region of the weights, the optimal solution is very sensitive to the weight selection: small changes in the weights change the solution. In the case of a large length of optimality interval or large optimality area, small changes in the weight values do not change the optimal solution, which indicates that the optimal solution is stable in such regions.

\section{Conclusions}

In this paper, a general decision making methodology is described and applied to find the best printed circuit board design in the presence of conflicting performance criteria. The method is known as the weighting method applied to normalized criteria. The methodology provides the basis for the complete characterization of the solution in terms of the weight selection. The numerical results indicate which alternatives can be optimal, give the conditions when they are optimal, and also show the stability regions of the results. In our analysis, the weighting method was selected because of its simplicity. However, there are several alternative methods known in literature which can also be used to solve this problem. Different approaches are based on different preferences and therefore they might lead to different solutions. The comparison of the available methods and method choice procedures are discussed for example, in [Szidarovszky et al 1986].

The methodology described in the paper can be used in all decision making problems when more than one criteria has to be taken into account. The uncertainty of the model parameters can be handled by stochastic simulation. The application of that methodology for printed circuit wire board design will be the subject of a future paper. 


\begin{tabular}{|c|c|c|c|}
\hline alternative & cost $(\$)$ & performance $(\exp 1 \mathrm{MHz})$ & reliability \\
\hline 1 & 84812.3 & 3.20 & 0.9999 \\
2 & 12957.4 & 4.46 & 0.9999 \\
3 & 7951.1 & 5.54 & 0.9999 \\
4 & 14842.1 & 6.49 & 0.9999 \\
5 & 23559.0 & 7.32 & 0.9999 \\
6 & 31804.6 & 8.06 & 0.9999 \\
7 & 39093.1 & 8.72 & 0.9999 \\
8 & 45416.4 & 9.32 & 0.9997 \\
9 & 50887.4 & 9.86 & 0.9994 \\
10 & 55636.1 & 10.03 & 0.9985 \\
11 & 59780.9 & 10.08 & 0.9958 \\
12 & 63421.0 & 11.20 & 0.9879 \\
13 & 66638.2 & 11.57 & 0.9637 \\
14 & 69498.9 & 11.92 & 0.8918 \\
\hline
\end{tabular}

Table 2: Optimal Decisions and Their Conditions

\begin{tabular}{|c|c|c|c|}
\hline $\begin{array}{l}\text { Optimal alternative } \\
\mathrm{r}\end{array}$ & $\begin{array}{l}\text { Left endpoint of } \\
\text { interval for } \alpha\end{array}$ & $\begin{array}{l}\text { Right endpoint of } \\
\text { interval for } \alpha\end{array}$ & Length of interval \\
\hline $3 \quad(s=4)$ & .539 & 1 & .461 \\
\hline $4 \quad(s=5)$ & .461 & .539 & .078 \\
\hline
\end{tabular}




\begin{tabular}{|l|l|l|l|}
\hline $15(\mathrm{~s}=16)$ & 0 & .461 & .461 \\
\hline
\end{tabular}

Figure 3: Optimal Decisions and Their Conditions

Table 3: Optimal Decisions and Their Conditions

\begin{tabular}{|l|l|l|}
\hline Optimal alternative $\mathrm{r}$ & Vertices of the simplex & Area of the simplex \\
\hline 3 & $(.5390, .4610),(0,0),(1,0)$ & .2305 \\
4 & $\begin{array}{l}(.5390, .4610),(.3442, .4229),(0, .0001), \\
(0, .0002), \quad .3866, .4721),(.4292, .5175),\end{array}$ & \\
& $(.4469, .5325),(.4608, .5391)$ \\
& $(.3442, .4229),(.0277, .0365),(0, .0003)$, \\
5 & $(.0269, .0355), \quad .0018$ \\
\end{tabular}




\begin{tabular}{|c|c|c|}
\hline & $(.0958, .1229),(.1867, .2355),(.3011, .373)$ & \\
\hline 6 & $\begin{array}{lll}(0, .0002), & (.0277, .0365), & (0, .0003), \\
(.0264, .0349),(0, .0007) & \end{array}$ & 0 \\
\hline 7 & $\begin{array}{lll}(.0269, .0355), & (.0264, .0349), & (0, .0007), \\
(0, .0020) & \end{array}$ & 0 \\
\hline 8 & $\begin{array}{l}(.0277, .0365), \\
(.0468, .0611), \\
(0, .0056)\end{array}$ & .0001 \\
\hline 9 & $\begin{array}{l}(.0468, .0611), \quad(.0958, .1229), \quad(0, .0056), \\
(0, .0169)\end{array}$ & .0006 \\
\hline 10 & $\begin{array}{l}(.0958, .1229), \quad(.1866, .2355), \quad(0, .0169), \\
(0, .0512)\end{array}$ & .0037 \\
\hline 11 & $\begin{array}{l}(.1867, .2355), \quad(.3011, .3733), \quad(0, .0512), \\
(0, .1495)\end{array}$ & .0171 \\
\hline 12 & $\begin{array}{l}(.3442, .4229), \\
(.3011, .3733),(0, .1495),(0, .39666), 4721)\end{array}$ & .0475 \\
\hline 13 & $\begin{array}{l}(.3866, .4721), \quad(.4292, .5175), \quad(0, .3674), \\
(0, .6527)\end{array}$ & .0677 \\
\hline 14 & $\begin{array}{lll}(.4292, .5175), & (.4469, .5325), & (0, .6527) \\
(0, .8455) & \end{array}$ & .0474 \\
\hline 15 & $\begin{array}{lll}(.4469, .5325), & (.4608, .5391), & (0, .8455) \\
(0,1)\end{array}$ & .0392 \\
\hline
\end{tabular}

\section{References}

Johnson, B. C., 1989, class notes of ECE 554, University of Arizona.

Mead, C. and Conway, L., 1980, Introduction to VLSI Systems. Addison-Wesley, Reading, Massachusetts.

Palusinski, O. A., 1997, class notes of ECE 654, University of Arizona

Smith, K., 1997, private communication

Szidarovszky,F., M. Gershon, L. Duckstein (1986) Techniques of Multi-objective Decision Making in Systems Management. Elsevier, Amsterdam. 
Tummala, R. R. and E. J. Rymaszewski (Eds.), 1992, Microelectronics Packaging

Figure 1: Price per square inch of printed wire boards with high-, medium-, and lowdensity lines [Johnson 1989] 